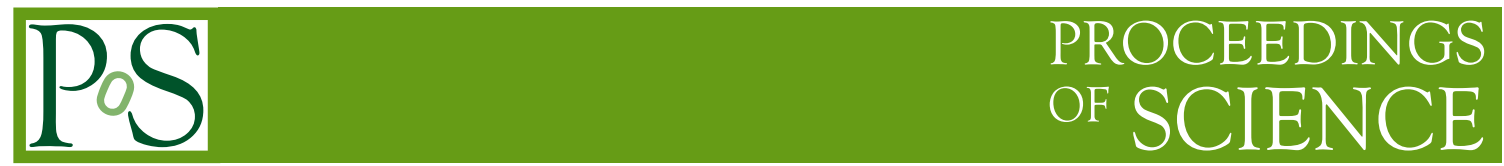

\title{
Search for jet quenching effects in high-multiplicity proton-proton collisions at $\sqrt{s}=13 \mathrm{TeV}$
}

\section{Kotliarov Artem* for the ALICE Collaboration}

Nuclear Physics Institute of the Czech Academy of Sciences, Rez, Czech Republic

E-mail: kotliarov@ujf.cas.cz

The ALICE Collaboration presents results of a search for jet quenching effects in high-particle multiplicity pp collisions at $\sqrt{s}=13 \mathrm{TeV}$. The analysis is based on the semi-inclusive acoplanarity spectrum of charged jets recoiling from a high- $p_{\mathrm{T}}$ trigger-track. Significant broadening and suppression of the recoil jet acoplanarity distribution is observed in high-multiplicity pp collisions in real data. However, similar features are also seen in events simulated by the PYTHIA 8 Monash Monte Carlo generator. The simulation reveals that the observed effects result from a bias induced by the ALICE high-multiplicity trigger, which enhances the probability to have a high- $p_{\mathrm{T}}$ recoil jet in the forward trigger detectors and which biases towards multi-jet final states.

The Ninth Annual Conference on Large Hadron Collider Physics - LHCP2021

7-12 June 2021

Online 


\section{Introduction}

Formation of quark-gluon plasma (QGP) in nucleus-nucleus collisions is accompanied by a strong collective flow of particles [1] and jet quenching [2]. Signatures of the collective flow were, however, observed in high-energy proton-proton (pp) collisions with large event activity [3]. This observation led to an active search for jet quenching in pp collisions, since measurements of jet quenching would serve as strong evidence for the QGP formation in small collision systems [4].

Jet quenching manifests itself as the modification of the jet shower due to a strong interaction of the partonic shower with the hot and dense medium. As a result of this interaction, the yield suppression of high- $p_{\mathrm{T}}$ hadrons or jets and medium-induced acoplanarity is observed. The suppression can be quantified by means of the nuclear modification factor, obtained as a ratio of the particle or jet yield measured in nucleus-nucleus collisions and the expected yield from a superposition of independent pp collisions. However, this approach cannot be utilized for jet quenching measurements in high-multiplicity pp collisions, since the Glauber scaling factor for the pp reference is undefined.

In these proceedings, we present the results of a novel approach to search for jet quenching effects in high-particle multiplicity pp collisions, which is based on measurements of the medium-induced acoplanarity [5] by means of the semi-inclusive distribution of charged-particle jets recoiling from a high- $p_{\mathrm{T}}$ trigger track (TT) [6]. The search for jet quenching is performed by comparing the spectra from minimum bias (MB) and high-multiplicity (HM) events.

\section{Analysis}

The analysis is based on data from pp collisions at $\sqrt{s}=13 \mathrm{TeV}$ measured by the ALICE detector [7] during the LHC Run 2. Events of interest were selected by means of the MB and HM online triggers. The collected data sets correspond to integrated luminosity $0.098 \mathrm{pb}^{-1}$ and $13 \mathrm{pb}^{-1}$, respectively. The triggers are based on signals measured by the V0A $(2.8<\eta<5.1)$ and V0C $(-3.7<\eta<-1.7)$ scintillator arrays [8]. The MB trigger required a time coincidence of the V0A and V0C signals, whereas the HM trigger selected events where the sum of both signal amplitudes, denoted as V0M, exceeded a given threshold. In the subsequent offline selection, event activity was further constrained to the range $5<\mathrm{V} 0 \mathrm{M} /\langle\mathrm{V} 0 \mathrm{M}\rangle<9$, where $\langle\mathrm{V} 0 \mathrm{M}\rangle$ denotes the mean V0M amplitude measured in MB events.

The selected charged tracks have pseudorapidity constrained to $|\eta|<0.9$ and transverse momentum $p_{\mathrm{T}}>0.15 \mathrm{GeV} / c$. These tracks were utilized to reconstruct charged-particle based anti- $k_{\mathrm{T}}$ jets with $R=0.4$. The reconstructed jets were required to be in the ALICE fiducial volume $\left|\eta_{\text {jet }}\right|<0.9-R$. Hadron-jet acoplanarity was studied with the semi-inclusive $\Delta_{\text {recoil }}$ observable [9], which removes the jet yield uncorrelated with the TT in a fully data-driven way. The $\Delta_{\text {recoil }}$ is defined as the difference between two per-trigger normalized yields of recoil jets associated with two exclusive trigger-track $p_{\mathrm{T}}$ classes, $\mathrm{TT}_{\mathrm{Sig}}$ and $\mathrm{TT}_{\mathrm{Ref}}$,

$$
\Delta_{\text {recoil }}\left(p_{\mathrm{T}, \text { Jet }}^{\text {ch,reco }}, \Delta \varphi\right)=\left.\frac{1}{N_{\text {trig }}} \frac{\mathrm{d}^{2} N_{\text {jets }}}{\mathrm{d} p_{\mathrm{T}, \text { jet }}^{\text {ch,rec }} \mathrm{d} \Delta \varphi}\right|_{p_{\mathrm{T}, \text { trig }} \in \mathrm{TT}_{\text {Sig }}}-\left.C_{\text {Ref }} \frac{1}{N_{\text {trig }}} \frac{\mathrm{d}^{2} N_{\text {jets }}}{\mathrm{d} p_{\mathrm{T}, \text { jet }}^{\text {ch,reco }} \mathrm{d} \Delta \varphi}\right|_{p_{\mathrm{T}, \text { trig }} \in \mathrm{TT}_{\text {Ref }}},
$$

where $N_{\text {trig }}$ is the number of TT in an event, $\Delta \varphi$ is the azimuthal angle between the TT and a recoil jet, and $C_{\mathrm{Ref}}$ is a correction factor close to 1 extracted from data [9]. The chosen trigger-track $p_{\mathrm{T}}$ 
intervals are $20<p_{\mathrm{T}}<30 \mathrm{GeV} / c$ and $6<p_{\mathrm{T}}<7 \mathrm{GeV} / c$ for $\mathrm{TT}_{\mathrm{Sig}}$ and $\mathrm{TT}_{\text {Ref }}$, respectively. Jet $p_{\mathrm{T}}$ was corrected for the median underlying event density $\rho$ following the procedure discussed in Ref. [10]. The corrected jet $p_{\mathrm{T}}$ is denoted as $p_{\mathrm{T}, \text { jet }}^{\text {ch,reco }}$.

\section{Results}

The left column of Fig. 1 compares uncorrected $\Delta_{\text {recoil }}(\Delta \varphi)$ spectra measured in the MB and $\mathrm{HM}$ events. Each figure corresponds to different jet $p_{\mathrm{T}}$ intervals. The acoplanarity spectra measured in the HM events exhibit marked suppression at $\Delta \varphi \approx \pi$ and broadening when compared to the corresponding spectra from the MB events. The observed modifications resemble jet quenching. Further, it was investigated whether the Monash tune [11] of the PYTHIA 8 event generator, which does not account for jet quenching, can reproduce the observed modifications, see the right column of Fig. 1. Qualitative comparison with the real data reveals that the PYTHIA acoplanarity spectra

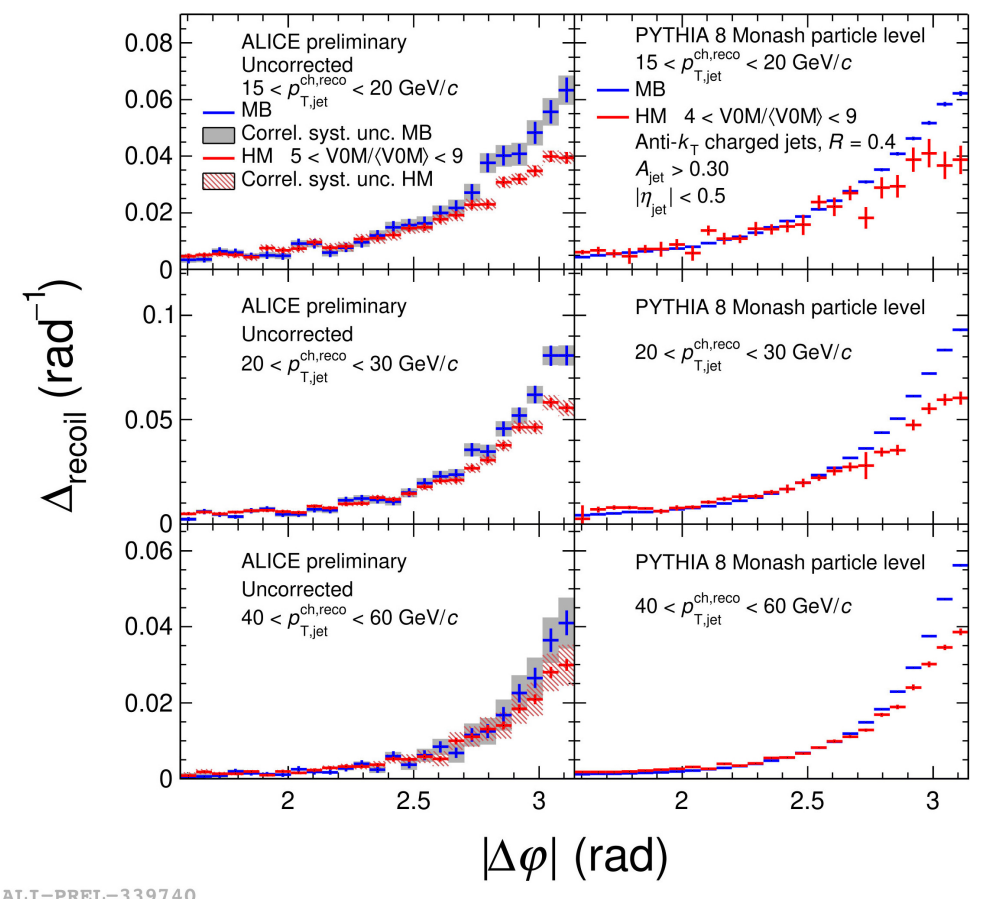

ALI-PREL-339740

Figure 1: Acoplanarity distributions for different jet $p_{\mathrm{T}, \mathrm{Jet}}^{\mathrm{ch} \text {,rec }}$ intervals in the MB and HM events. Left and right columns show the uncorrected data and PYTHIA 8 Monash particle level ones, respectively.

exhibit similar features. Therefore, the observed modifications are not caused by jet quenching. The PYTHIA 8 event generator was further used to search for the origin of these phenomena. The left panel of Fig. 2 shows a pseudorapidity distribution of recoil jets with $p_{\mathrm{T}, \text { jet }}^{\mathrm{ch}}>25 \mathrm{GeV} / c$ as the function of event activity. One can see that the probability to observe a high- $p_{\mathrm{T}}$ recoil jet in the V0 detectors grows when the event multiplicity increases. Less enhancement is seen in the V0A acceptance, since the V0A detector is placed farther from the interaction point [8].

In addition, the right panel of Fig. 2 compares probability distributions to find in a HM or MB event with $\operatorname{TT}\{20,30\}$ a given number of recoil jets with $p_{\mathrm{T}, \mathrm{jet}}^{\mathrm{ch}}>25 \mathrm{GeV} / c$, which are in the ALICE fiducial volume. Both spectra are steeply falling. Their ratio, which is shown in the 

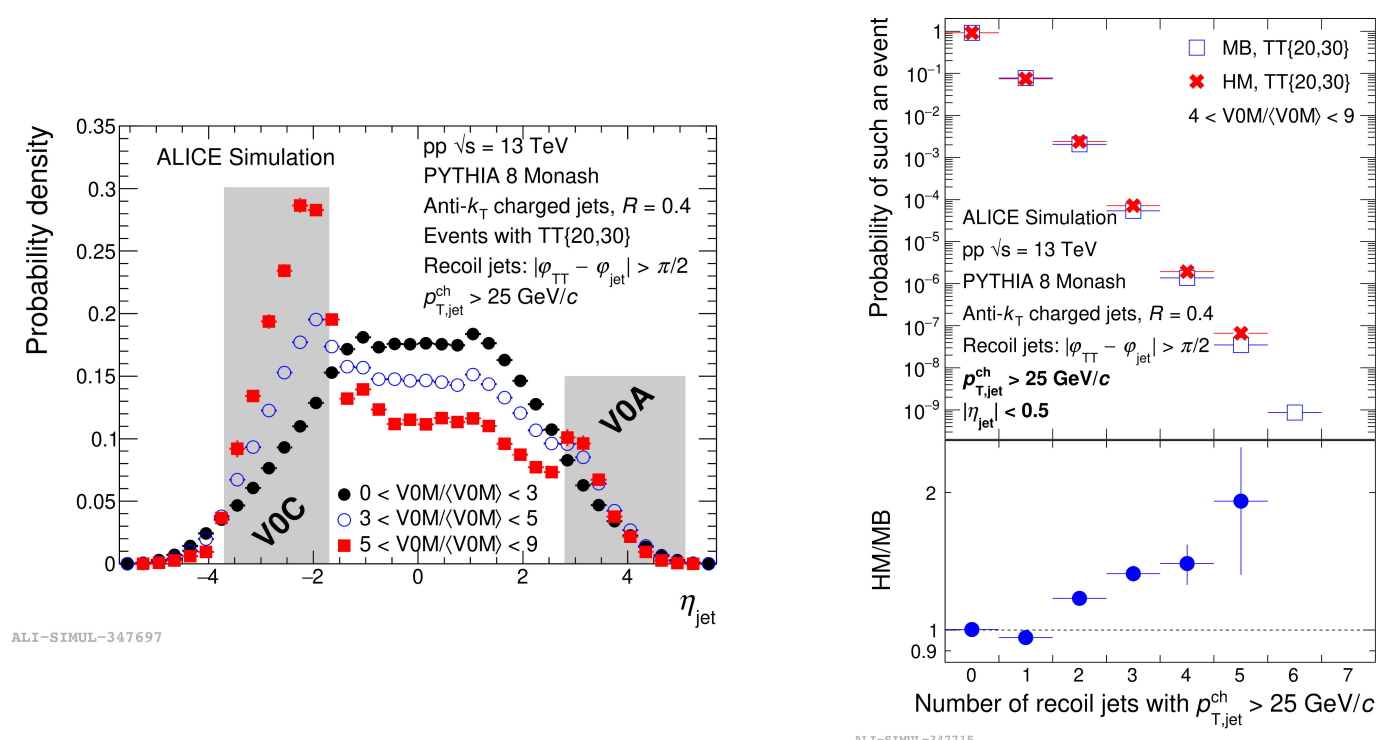

Figure 2: Left: Pseudorapidity distribution of recoil jets with $p_{\mathrm{T}, \mathrm{jet}}^{\mathrm{ch}}>25 \mathrm{GeV} / c$ in events with $\mathrm{TT}\{20,30\}$ for different event activity bias in terms of $\mathrm{VOM} /\langle\mathrm{V} 0 \mathrm{M}\rangle$. The gray boxes represent the V0A and V0C acceptances. Right: Probability density distribution of the number of recoil jets with $p_{\mathrm{T}, \mathrm{jet}}^{\text {ch }}>25 \mathrm{GeV} / c$ in MB and HM events with TT $\{20,30\}$. Bottom panel shows HM/MB ratio of the probability density functions.

bottom panel, reveals that the relative abundance of events with a single high- $p_{\mathrm{T}}$ recoil jet is lower for the HM selection when compared to the MB one. This single recoil jet is missed in the ALICE central barrel, therefore it can induce the suppression of the acoplanarity distribution (Fig. 1). Besides that, the HM events have enhanced probability for a final state with multiple recoil jets. This result is consistent with the observed broadening, since a multi-jet topology has on average greater acoplanarity.

\section{Conclusion}

The first semi-inclusive measurements of the recoil jet acoplanarity have been performed in the $\mathrm{HM}$ and MB pp collisions at $\sqrt{s}=13 \mathrm{TeV}$. The obtained results show marked suppression and broadening of the HM acoplanarity distributions compared to the MB ones. The observed modifications can, however, be reproduced by the PYTHIA 8 event generator. The PYTHIA simulations revealed that the HM trigger increases the probability to observe a high- $p_{\mathrm{T}}$ recoil jet in the acceptance of the V0 detectors. Moreover, the HM trigger biases toward events with multiple recoil jets in a final state that lead to an increase of hadron-jet acoplanarity which obscures any possible jet quenching signal.

Acknowledgments: This work was supported by the Ministry of Education, Youth and Sports of the Czech Republic project LTT17018.

\section{References}

[1] U. Heinz and R. Snellings, Collective flow and viscosity in relativistic heavy-ion collisions, Ann. Rev. Nucl. Part. Sci. 63 (2013), 123-151. 
[2] K. M. Burke et al., (JET Collaboration), Extracting the jet transport coefficient from jet quenching in high-energy heavy-ion collisions, Phys. Rev. C 90 (2014), 014909.

[3] V. Khachatryan et al., (CMS Collaboration), Observation of Long-Range Near-Side Angular Correlations in Proton-Proton Collisions at the LHC, JHEP 09 (2010), 091.

[4] B. Nachman, L. Mangano, Observables for possible QGP signatures in central pp collisions, CERN-TH-2017-184, arXiv:1708.08369 [hep-ph].

[5] L. Chen, G. Y. Qin, S. Y. Wei, B. W. Xiao and H. Z. Zhang, Probing Transverse Momentum Broadening via Dihadron and Hadron-jet Angular Correlations in Relativistic Heavy-ion Collisions, Phys. Lett. B 773 (2017), 672-676.

[6] L. Adamczyk et al., (STAR Collaboration), Measurements of jet quenching with semi-inclusive hadron+jet distributions in Au+Au collisions at $\sqrt{s_{\mathrm{NN}}}=200 \mathrm{GeV}$, Phys. Rev. C 96 (2017), 024905.

[7] K. Aamodt et al., [ALICE], The ALICE experiment at the CERN LHC, JINST 3 (2008), S08002.

[8] E. Abbas et al., [ALICE], Performance of the ALICE VZERO system, JINST 8 (2013), P10016.

[9] J. Adam et al., [ALICE], Measurement of jet quenching with semi-inclusive hadron-jet distributions in central Pb-Pb collisions at $\sqrt{s_{\mathrm{NN}}}=2.76 \mathrm{TeV}$, JHEP 09 (2015), 170.

[10] B. Abelev et al., [ALICE], Measurement of Event Background Fluctuations for Charged Particle Jet Reconstruction in Pb-Pb collisions at $\sqrt{s_{\mathrm{NN}}}=2.76 \mathrm{TeV}$, JHEP 03 (2012), 053.

[11] Skands, P., Carrazza, S. and Rojo J., Tuning PYTHIA 8.1: the Monash 2013 Tune, CERN-PHTH-2014-069, arXiv:1404.5630 [hep-ph]. 